\begin{tabular}{|c|l|}
\hline Title & Study on phase transition of Pb-Bi eutectic all loy by neutron transmission spectroscopy \\
\hline Author(s) & Takada, Osamu; Kamiyama, Takashi; Kiyanagi, Y oshiaki \\
\hline Citation & $\begin{array}{l}\text { Journal of Nuclear Materials, 398(1-3), 129-131 } \\
\text { https://doi.org/10.1016/.jnucmat.2009.10.022 }\end{array}$ \\
\hline Issue Date & 2010-03 \\
\hline Doc URL & http://hdl.handle.net/2115/43026 \\
\hline Type & article(author version) \\
\hline File Information & JNM398_1-3_129-131.pdf \\
\hline
\end{tabular}

Instructions for use 


\title{
Study on Phase Transition of Pb-Bi Eutectic Alloy by Neutron Transmission Spectroscopy
}

\author{
Osamu Takada* Takashi Kamiyama Yoshiaki Kiyanagi
}

Graduate school of engineering, Hokkaido University

Corresponding author: tel +81-11-706-7896, fax +81-11-706-7896,

Email: o-takada@eng.hokudai.ac.jp.

\begin{abstract}
LBE (Lead Bismuth Eutectic), which is a candidate for spallation targets, has some problems. One of them is expansion after solidification, which may bring about serious damage of an LBE loop. Two solid phases of $\beta$-phase (inter-metallic compound) and $\gamma$-phase (solid solution of $\mathrm{Pb}$ in $\mathrm{Bi}$ ) appear in $\mathrm{LBE}$, and it was reported that an increase of the $\gamma$-phase observed by X-ray corresponds to the volume expansion of LBE. We performed neutron spectroscopic transmission experiments using a pulsed neutron source in order to observe crystal transformation depending on the position and time for slow and rapid cooling LBE samples. It was found that the crystal structure of the slow cooling LBE varied depending on position while it was rather homogeneous for the rapid cooling sample. The re-crystallization of the $\gamma$-phase was more clearly observed for the rapid cooling sample than the slow one. We were able to obtain information of re-crystallization over the whole volume of the samples.
\end{abstract}

\section{Introduction}

Lead Bismuth Eutectic (LBE) is a candidate for spallation targets and coolant in ADS (Accelerator Driven System) because LBE is inert against air, and has low slowing down power for neutrons. However, LBE has some problems, such as generation of Po, corrosion of 
steel and expansion after solidification. The expansion may causes a serious problem, since rupture of coolant tubes may occur if liquid LBE solidify fortuitously.

Solid LBE has two solid phases, $\beta$-phase (intermetallic compound) and $\gamma$-phase (solid solution of $\mathrm{Pb}$ in $\mathrm{Bi}$ ). $\beta$-phase has hcp structure and $\gamma$-phase has rhombohedral structure. Expansion results from phase transformation ( $\beta$ to $\gamma$ ), since the rhombohedral structure of the $\gamma$-phase occupies more space than the hcp structure.

H. Grasbrenner et al. investigated the expansion of LBE by various methods, glass vial break test, X-ray diffraction (XRD), dilatometer measurement and so on [1]. They showed a relation between cooling speed and expansion, and also a relation between the $\gamma$-phase growth and the expansion by XRD. However, the structural data obtained by the XRD measurement were confined to the surface because of the heavy metals. Therefore, we intend to study the volumetric structure change of LBE depending on position to understand the $\gamma$-phase transformation in more detail.

Neutron transmission is suitable for this purpose, since neutrons have strong penetration power and the transmission cross-section includes information on crystal texture [2,3]. Therefore, we adopted neutron transmission spectroscopy to observe LBE crystal texture.

Here, we report position and time dependent volumetric changes of crystal structure for slow and rapid cooling LBE samples observed by a pulsed neutron transmission spectroscopy.

\section{Experimental}

Neutron transmission spectroscopy combined with a position sensitive detector is useful to measure position dependent structural information of materials especially composed of heavy metals like LBE, while X-ray only see surface of such materials. By using a pulsed neutron source combined with wavelength analysis using the time of flight, we get a wavelengthspectrum from a moderator (incident beam, $I_{\text {incident }}(\lambda)$ ) and a spectrum transmitted through

an object, $I_{\text {transmitted }}(\lambda)$. The transmission cross-section of materials can be calculated by an equation,

$$
\Sigma=-\ln \left(I_{\text {transmitted }}(\lambda) / I_{\text {incident }}(\lambda)\right) / a
$$

Here, $a$ is the thickness of the material and $\lambda$ is neutron wavelength. Crystal texture and structure information are obtained by analyzing Bragg edges appearing in the total crosssection over a few angstroms of neutron wavelength, which correspond to the maximum lattice planes $d_{\max }$ with a relation of $2 d_{\max }=\lambda$. The shape around a Bragg edge depends not only on crystal structure but also on the crystalline distribution. The advantage of this method 
is that it can give crystal structural information inside materials and position dependent changes at once by using a 2-dimentional position sensitive detector (2D-PSD).

Measurements with neutron transmission spectroscopy were carried out at the pulsed cold neutron source at the $45 \mathrm{MeV}$ electron linac facility at Hokkaido University. We used a Liglass 2D-PSD having $16 \times 16=256$ pixels with about $2.1 \mathrm{~mm} \times 2.1 \mathrm{~mm}$ pixel size [4]. The efficiency of this detector is almost $100 \%$ for cold neutrons. This detector was placed close behind the object. The neutron wavelength was analyzed by the time-of-flight method, $t=L / v$, which is proportional to the neutron wavelength $\lambda$. Here, $L$ is flight-path length, $t$ flight time and $v$ velocity of the neutron.

The formation of texture in LBE is dependent on the cooling rate from liquid to solid state. So, we prepared two types of LBE samples processed by different cooling methods. One is rapid cooling (water cooling) and the other slow cooling (cooling in the furnace). First, LBE chips were loaded into a mold and kept at $150^{\circ} \mathrm{C}$ for 30 minutes in an electric furnace. Thereafter one was cooled down to room temperature in the furnace (slow cooling), and the other cooled down in ice water (rapid cooling). The slow cooling rate was $0.02 \mathrm{~K} / \mathrm{s}$ in opposition to the rapid cooling rate of approximately $30 \mathrm{~K} / \mathrm{s}$. The molded sample size was 20 mm width, $20 \mathrm{~mm}$ height and $9 \mathrm{~mm}$ thickness. We observed crystal phase and structure changes for these samples as a function of elapsed time.

\section{Results and discussion}

Measurements on two LBE samples have been carried out 4 times so far: after solidification (called 'fresh' after now), at 2-weeks later (2weeks), 3-months (3mth), and 5months (5mth).

In order to see changes of the crystal texture depending on position, we deduced the total cross-section at each pixel. Figure 1 shows examples of the data of the fresh slow cooling

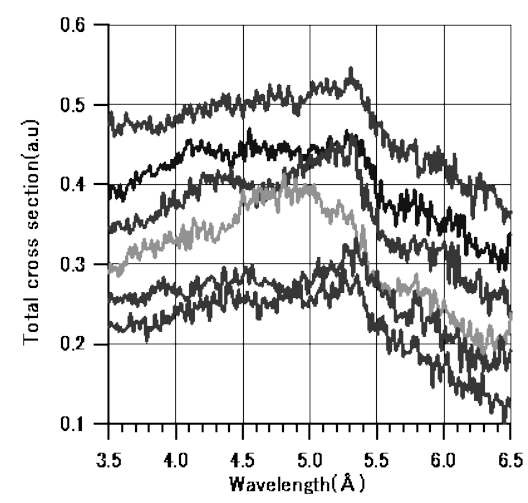

Fig.1 Examples of total crosssection depending on the position in fresh slow cooling LBE.

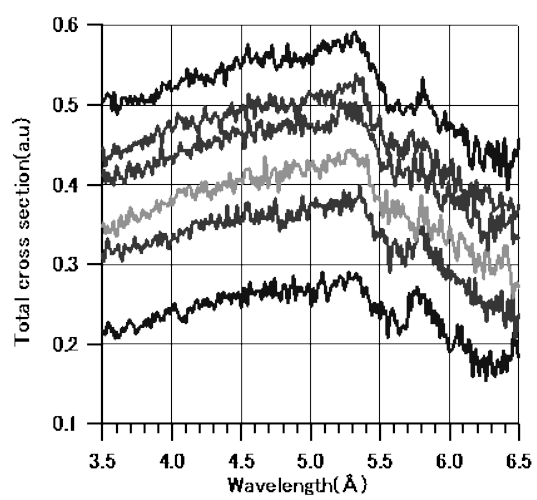

Fig.2 Examples of total crosssection depending on the position in fresh rapid cooling LBE. 
LBE (not absolute values). The total cross-section varies pixel by pixel, which indicates that the crystal structure is nonuniform in the sample volume. The cross-section differences would be attributed to the orientational anisotropy of the crystallites of the $\beta$ and $\gamma$-phases and also to the different crystal growth. Fig.2 shows position dependent total cross-sections of the fresh rapid cooling LBE. The shapes are not so different from each other, which suggests that the rapid cooling procedure make the crystal structure rather uniform over the sample. In the measurement, the slow cooling LBE sample covered 30 pixels of 2D-PSD, and the rapid cooling one covered 36 pixels. In the slow cooling LBE, the edge of $\mathrm{Bi}(012)$ at $6.5 \AA$ was observed in roughly 20 pixels right after solidification, and then the number increased to 25 pixels at 5-months data. On the other hand, in the rapid cooling one, the edge was observed in 27 pixels right after solidification, and increased to 36 pixels (all pixels) at 5-months one. This indicates that the precipitation in the rapid cooling LBE started earlier than the slow cooling one. However, no systematic trend of $\gamma$-phase precipitation depending on the position was found in our measurements.

Figure 3 shows time-dependent changes of the shapes of the total cross-sections (not absolute values) of the slow cooling LBE, and Fig. 4 that of the rapid cooling LBE. These were data integrated over all pixels of the 2D-PSD covering the samples. Small humps gradually appeared with time around $4.6 \AA$, corresponding to the Bragg edges of the $\gamma$-phase at $4.55 \AA$ (Bi (110)) and 4.74§ (Bi (104)), which are clearly recognized especially in the 5months data. An increase of the peak at $6.5 \AA$, Bragg edge of $\mathrm{Bi}(012)$, is clearly observed in both samples.

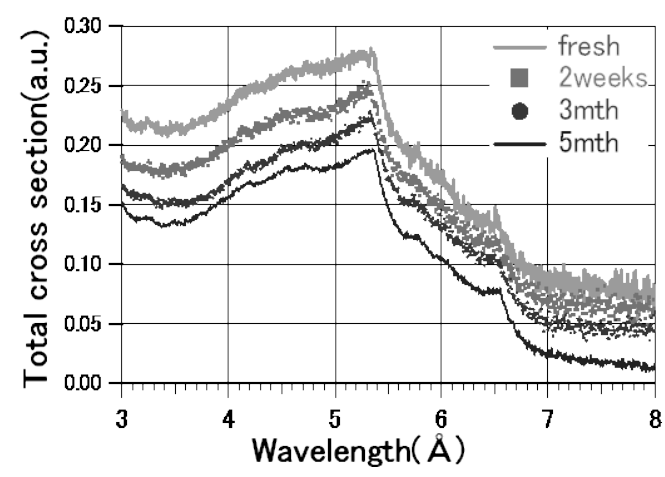

Fig.3 Changes of total cross-sections of slow cooling LBE.

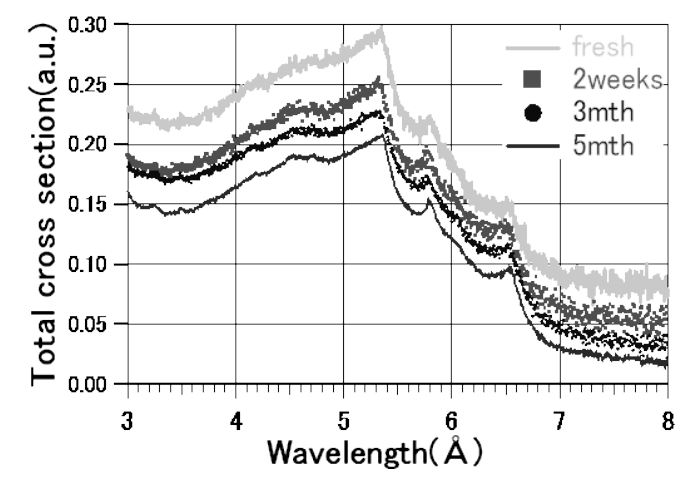

Fig.4 Changes of total crosssections of rapid cooling LBE.

The $6.5 \AA$ peak of $\operatorname{Bi}(012)$ is isolated. So, we tried to estimate $\gamma$-phase transformation by analyzing this peak, since LBE phase transformation will be proportional to the cross-section value. Fig.5 shows the cross-section around the Bragg edge of $\operatorname{Bi}(012)$. The quantitative estimation process was as follows; fit a linear function to the cross-section of $\mathrm{Bi}(012)$, draw a base line shown in Fig.5, and then extract the cross-section of $\mathrm{Bi}(012)$ from the cross-section between the curve and the line. The integrated intensity of this cross-section was evaluated 
for each cross-section datum. Fig. 6 shows integrated cross-section changes of the Bragg edge with time. Although the results were obtained from poor statistics data, gradual increase of $\gamma$-phase with time for both LBE samples was clearly seen. Sharp increase is observed between the fresh and 2-weeks data; however, the rapid cooling one seems to be steeper than that of the slow cooling one. The amount is much higher in rapid cooling LBE than that in slow one.

\section{Conclusions}

We studied the phase transition of LBE by the neutron transmission method, and succeeded in getting crystal information of LBE over sample volume depending on the position and the time. The crystal structure was more uniform in the rapid cooling sample than in the

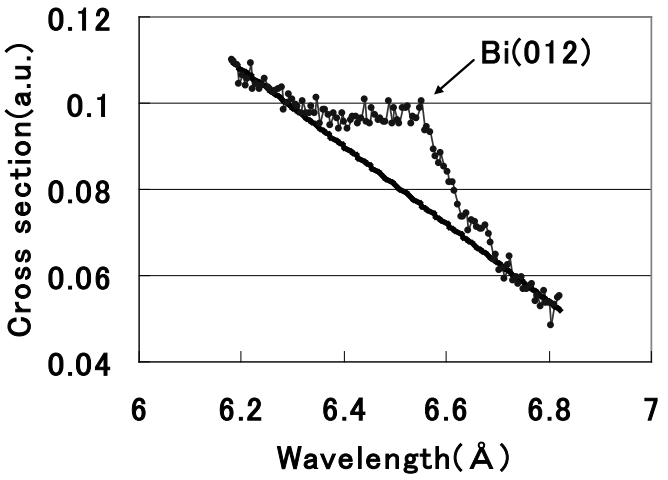

Fig.5 Bragg edge of $\mathrm{Bi}(012)$. The solid line is a base line coming from other contributions.

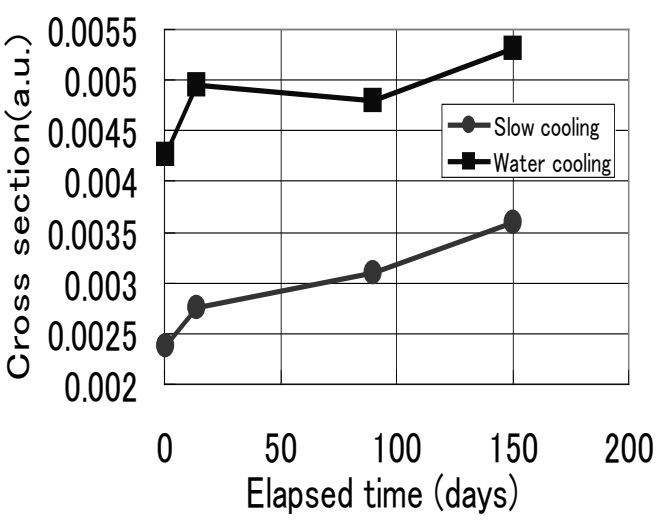

Fig.6 Time dependent cross-section change of the Bragg edge of $\operatorname{Bi}(012)$. slow cooling one. The estimated value of the $\gamma$-phase precipitation in the rapid cooling sample was larger than in the slow cooling one, which is consistent with previous results [1].

Here, we only examined two types of LBE samples, and the data were limited to short periods. To find the optimum cooling method avoiding rapid expansion, we will need more detailed information on the precipitation process for more samples with different cooling speeds.

\section{References}

[1] H. Grasbrenner, F. Gröschel, H. Grimmer, J. Patorski, and M. Rohde, Journal of Nuclear Materials, 343 (2005) 341-348.

[2] Y. Kiyanagi, T. Kamiyama, T. Nagata, F. Hiraga, S. Suzuki, Physica B, 385-386(2006) 930-932.

[3] Y. Kiyanagi, T. Kamiyama, T. Nagata, F.Hiraga, Key Engineering Material, Vols. 321323 (2006) 1663-1666.

[4] K. Mizukami, S. Sato, H. Sagehashi, S. Ohnuma, M. Ooi, H. Iwasa, F. Hiraga, T. Kamiyama and Y. Kiyanagi, Nucl. Instr. and Meth. in Physics Research A, 529 (2004) 310-312. 